\title{
Diabatic description of bottomoniumlike mesons
}

\author{
R. Bruschini $\oplus^{1, *}$ and P. González $\oplus^{1,2, \uparrow}$ \\ ${ }^{1}$ Unidad Teórica, Instituto de Física Corpuscular (Universidad de Valencia-CSIC), \\ E-46980 Paterna (Valencia), Spain \\ ${ }^{2}$ Departamento de Física Teórica, Universidad de Valencia, E-46100 Burjassot (Valencia), Spain
}

(Received 11 May 2021; accepted 18 May 2021; published 17 June 2021)

\begin{abstract}
We apply the diabatic approach, specially suited for a QCD based study of conventional (quarkantiquark) and unconventional (quark-antiquark + meson-meson) meson states, to the description of hidden-bottom mesons. A spectral analysis of the $I=0, J^{++}$and $1^{--}$resonances with masses up to about $10.8 \mathrm{GeV}$ is carried out. Masses and widths of all the experimentally known resonances, including conventional and unconventional states, can be well reproduced. In particular, we predict a significant $B \bar{B}^{*}$ component in $\Upsilon(10580)$. We also predict the existence of a not yet discovered unconventional $1^{++}$narrow state, with a significant $B_{s} \bar{B}_{s}^{*}$ content making it to decay into $\Upsilon(1 S) \phi$, whose experimental discovery would provide definite support to our theoretical analysis.
\end{abstract}

DOI: $10.1103 /$ PhysRevD.103.114016

\section{INTRODUCTION}

The unified description of conventional and unconventional heavy-quark mesons from QCD, the strong interaction theory, is a current theoretical challenge in hadron physics. Due to the current impossibility of solving QCD in the nonperturbative regime, effective field theories directly connected to QCD, involving quark and gluons or hadrons as degrees of freedom, have been developed for the study of the heavy-quark meson structure, see for instance [1] and references therein. On the other hand, QCD calculations of heavy-quark mesons in the lattice have been performed. These comprise quenched analyses involving $Q \bar{Q}(Q$ : heavy quark, $b$ or $c$ ) with gluons as the light field [2,3], and unquenched studies with $Q \bar{Q}$ and meson-meson components incorporating also light sea quarks in the light field [4-6]. A nice feature of the lattice, concerning phenomenology, is that it provides a straightforward way to compute complete heavy-quark meson potentials from QCD: the static light field energies evaluated in lattice are related to static potentials. More concretely, following a Born-Oppenheimer approximation quenched static energies can be directly identified with potentials in a Schrödinger equation for $Q \bar{Q}$, see for instance [2,7]. This allows for a QCD-based description of conventional

\footnotetext{
*oberto.bruschini@ific.uv.es

pedro.gonzalez@uv.es
}

Published by the American Physical Society under the terms of the Creative Commons Attribution 4.0 International license. Further distribution of this work must maintain attribution to the author(s) and the published article's title, journal citation, and DOI. Funded by SCOAP ${ }^{3}$. quarkonium $(b \bar{b}$ or $c \bar{c})$ in terms of a potential whose spinindependent part corresponds to a Cornell (funnel) form. As for unquenched static energies, calculated for $Q \bar{Q}$ in the presence of meson-meson configurations, the BornOppenheimer approximation, which is a single channel one, is not valid anymore. Instead, a diabatic approach [8] permits their connection with the potential matrix in a multichannel Schrödinger equation for the $Q \bar{Q}$ and mesonmeson components.

Strictly speaking the static potential is only exact in the limit of infinite heavy-quark mass. For bottomonium $(b \bar{b})$ with a quark mass, $m_{b}$, much larger than the QCD scale, $\Lambda_{\mathrm{QCD}}$, the static limit represents a rather good approximation. For charmonium $(c \bar{c})$, with a much lower quark mass, $m_{c}$, nonstatic contributions could be significant. Despite this drawback, in the last two decades, much more attention has been paid to the theoretical description of the excited spectrum of charmonium, the reason being the discovery, starting at 2003 with the $\chi_{c 1}(3872)$, of charmoniumlike mesons whose properties (masses and widths) cannot be properly described from a conventional $c \bar{c}$ structure. The role played by explicit or implicit open charm mesonmeson components in the description of these unconventional states has been recognized, and alternative models (meson-meson molecules, tetraquarks, hadrocharmonium) have been formulated, some reviews are [9-13].

Quite recently, a (nonperturbative) diabatic description of the $I=0, J^{++}$and $1^{--}$hidden-charm mesons with masses up to about $4 \mathrm{GeV}$, in terms of $c \bar{c}$ and meson-meson components, has been undertaken $[8,14]$. A major difference with respect to other nonperturbative studies involving the same degrees of freedom, see for example $[15,16]$, is the incorporation of a lattice-based form of the mixing 
potential instead of an ansatz with no clear connection to QCD. Despite the dearth of lattice data, and the technical approximations followed for tackling the diabatic equations, the results obtained (masses and widths) are encouraging. This supports the diabatic approach in QCD as an appropriate framework for a unified and complete nonperturbative description of conventional and unconventional heavy-quark mesons.

For hidden-bottom mesons there have been in the past many speculations about possible bottomoniumlike partners of the unconventional charmoniumlike states, see for instance [17] and references therein. The partner hypothesis is based on the consideration that hidden-charm and hidden-bottom mesons can be described from the same flavor independent static potential (up to a constant). This is clearly acceptable for (conventional) charmonium, $c \bar{c}$, and bottomonium, $b \bar{b}$, with masses lying below the first openflavor meson-meson threshold, which are quite successfully described from a quark-antiquark Cornell potential. However, for unconventional states involving $Q \bar{Q}$ and open-flavor meson-meson components as well, the partner hypothesis is questionable, for it is doubtful that the offdiagonal terms in the static potential matrix, giving account of the $Q \bar{Q}$ and meson-meson mixings, be flavor independent. Experimentally, the situation is not well established due to the current dearth of data (masses and widths) for $I=0, J^{++}$hidden-bottom mesons above the first $\left(0^{++}\right)$ open-bottom meson-meson threshold, and the absence of data for $I=0,1^{--}$resonances with masses above the first $1^{--} S$-wave meson-meson threshold. From the theoretical point of view, the diabatic approach, generating the static potential matrix from lattice QCD data, can be an ideal tool to definitely settle this issue. Indeed, lattice data for the energy of static $b$ and $\bar{b}$ sources, when the $b \bar{b}$ configuration mixes with one or two open-flavor meson-meson ones, are available. From them a direct parametrization of the diabatic potential matrix is possible, and a QCD based prediction of the unknown excited spectrum is feasible. Actually, the hidden-bottom meson spectrum has been partially explored recently in a simplified diabatic treatment of $I=0,0^{-+}$and $1^{--}$resonances, involving only one $b \bar{b}$ channel and at most two distinct meson-meson thresholds masses $[18,19]$.

In this article we center on the diabatic description of hidden-bottom mesons. The main differences with respect to $[18,19]$ are (i) the consideration of all possible $b \bar{b}$ channels and all meson-meson threshold masses contributing, (ii) the mixing potential which in our case does not contain any short range (light quark meson exchange) contribution, in line with the use of constant mesonmeson potentials, and (iii) the use of a bound-state based approximation instead of a S-matrix approach to the spectral solutions. We restrict our study to $I=0, J^{++}$, and $1^{--}$resonances with masses up to about $10.8 \mathrm{GeV}$, two hundred $\mathrm{MeV}$ below the first $1^{--} S$-wave mesonmeson thresholds. Thus, as all the lower thresholds are known and have very small widths we avoid the uncertainty deriving from the partial knowledge of a threshold and the complexity due to possible threshold width effects. For the sake of technical simplicity in the evaluation of observables, we follow a two step description of resonances: first we approximate them by stable bound states incorporating closed meson-meson channels, and second we calculate mass corrections and widths from open meson-meson channels. We show that a fairly good description of the currently known $\mathrm{J}^{++}$and $1^{--}$experimental resonances in the realm of energy under study comes out. We predict that all these resonances except $\Upsilon(10580)$ have a very predominant $b \bar{b}$ component. For $\Upsilon(10580)$ the reduced, albeit dominant, $b \bar{b}$ probability allows us to give accurate account of leptonic width data. As for the not yet discovered resonances we predict that only for the third excited $1^{++}$ state there is a significant meson-meson component. Although not dominant, this component points out to $\Upsilon(1 S) \phi$ as a favored decay channel what could be relevant for its experimental discovery. Altogether these results indicate that a partner picture of hidden-charm and hidden-bottom mesons should be discarded once mesonmeson contributions start to play some role.

These contents are organized as follows. In Sec. II a brief review of the diabatic formalism particularized for hiddenbottom mesons is presented, and the diabatic potential matrix is built from lattice data. As an improvement over the previous development for hidden-charm mesons a distinctive treatment of hidden-strange thresholds is incorporated. In Sec. III the nonperturbative description of hidden-bottom mesons is done in two steps: first, a bound state approximation incorporating closed meson-meson thresholds is followed, and second, mass shifts and widths from open meson-meson thresholds are calculated. Finally, in Sec. IV our main results and conclusions are summarized.

\section{DIABATIC FORMALISM FOR HIDDEN-BOTTOM MESONS}

The diabatic approach in QCD has been developed in [8]. Hidden-bottom meson states with quantum numbers $J^{\mathrm{PC}}$, made of $b \bar{b}$ and open-bottom meson-meson $M_{1}^{(i)} \bar{M}_{2}^{(i)}$ components, with $M_{1}\left(\bar{M}_{2}\right)$ containing $q \bar{b}(\bar{q} b)$ where $q$ stands for a light quark, $q=u, d, s$, are solutions of the multichannel Schrödinger equation

$$
(\mathrm{K}+\mathrm{V}(\boldsymbol{r})) \Psi(\boldsymbol{r})=E \Psi(\boldsymbol{r})
$$

where $\Psi(\boldsymbol{r})$ is a column vector 


$$
\Psi(\boldsymbol{r})=\left(\begin{array}{c}
\psi_{b \bar{b}}(\boldsymbol{r}) \\
\psi^{(1)}(\boldsymbol{r}) \\
\vdots \\
\psi^{(N)}(\boldsymbol{r})
\end{array}\right)
$$

with $\psi_{b \bar{b}}(\boldsymbol{r})$ standing for the $b \bar{b}$ component, and $\psi^{(i)}(\boldsymbol{r})$, $i=1,2 \ldots$ for the $M_{1}^{(i)} \bar{M}_{2}^{(i)}$ component.

$\mathrm{K}$ is the kinetic energy matrix

$$
\mathrm{K}=\left(\begin{array}{cccc}
-\frac{1}{2 \mu_{b \bar{b}}} \nabla^{2} & & & \\
& -\frac{1}{2 \mu^{(1)}} \nabla^{2} & & \\
& & \ddots & \\
& & & -\frac{1}{2 \mu^{(N)}} \nabla^{2}
\end{array}\right)
$$

where $\mu_{b \bar{b}}$ is the reduced $b \bar{b}$ mass, $\mu^{(i)}$ is the reduced $M_{1}^{(i)} \bar{M}_{2}^{(i)}$ mass, and matrix elements equal to zero are not displayed.

$\mathrm{V}(\boldsymbol{r})$ is the diabatic potential matrix. Up to spin dependent terms that we shall not consider it can be formally written as

$$
\left(\begin{array}{cccc}
V_{\mathrm{C}}(r) & V_{\text {mix }}^{(1)}(r) & \cdots & V_{\text {mix }}^{(N)}(r) \\
V_{\text {mix }}^{(1)}(r) & T^{(1)} & & \\
\vdots & & \ddots & \\
V_{\text {mix }}^{(N)}(r) & & & T^{(N)}
\end{array}\right)
$$

where the diagonal elements $V_{\mathrm{C}}(r)$ and $T^{(i)}$ correspond to the $b-\bar{b}$ and $M_{1}^{(i)}-\bar{M}_{2}^{(i)}$ potentials respectively, and $V_{\text {mix }}^{(i)}(r)$ to the $M_{1}^{(i)} \bar{M}_{2}^{(i)}-b \bar{b}$ interaction potential.

More precisely, we express the $b \bar{b}$ component as

$$
\psi_{b \bar{b}}(\boldsymbol{r})=\sum_{t} R_{t}^{(0)}(r) \mathcal{Y}_{l_{t}^{(0)}, s_{t}^{(0)}}^{J, m_{J}}(\hat{\boldsymbol{r}})
$$

where the sum over $t$ goes from 1 to the number of pairs $\left(l_{b \bar{b}} \equiv l^{(0)}, s_{b \bar{b}} \equiv s^{(0)}\right)$ coupling to $J^{\mathrm{PC}}, R_{t}^{(0)}(r)$ stands for a radial wave function and

$$
\mathcal{Y}_{l, s}^{J, m_{J}}(\hat{r}) \equiv \sum_{m_{l}, m_{s}} C_{l, s, J}^{m_{l}, m_{s}, m_{J}} Y_{l}^{m_{l}}(\hat{\boldsymbol{r}}) \xi_{s}^{m_{s}}
$$

for an angular-spin wave function $(C$ is a Clebsch-Gordan coefficient, $Y_{l}^{m_{l}}$ a spherical harmonic, and $\xi_{s}^{m_{s}}$ a spin vector), and the $M_{1}^{(i)} \bar{M}_{2}^{(i)}$ component as

$$
\psi^{(i)}(\boldsymbol{r})=\sum_{k} R_{k}^{(i)}(r) \mathcal{Y}_{l_{k}^{(i)}, s_{k}^{(i)}}^{J, m_{J}}(\hat{\boldsymbol{r}})
$$

where the sum over $k$ goes from 1 to the number of pairs $\left(l^{(i)} \equiv l_{M_{1}^{(i)} \bar{M}_{2}^{(i)}}, s^{(i)} \equiv s_{M_{1}^{(i)} \bar{M}_{2}^{(i)}}\right)$ coupling to $J^{\mathrm{PC}}$.

(Let us note that we have changed the notation for the radial wave function with respect to our previous papers $[8,14]$. Here we use the standard $R$ and reserve $u$ for the reduced radial wave function, see next.)

Then, one has

$$
\int \mathrm{d} \Omega \psi_{b \bar{b}}^{*}(\boldsymbol{r}) \mathrm{V}(\boldsymbol{r}) \psi_{b \bar{b}}(\boldsymbol{r})=\sum_{t} R_{t}^{(0) *}(r) V_{\mathrm{C}}(r) R_{t}^{(0)}(r)
$$

$$
\begin{aligned}
& \int \mathrm{d} \Omega \psi^{(i) *}(\boldsymbol{r}) \mathrm{V}(\boldsymbol{r}) \psi_{b \bar{b}}(\boldsymbol{r})=\sum_{k, t} R_{k}^{(i) *}(r) V_{\text {mix }}^{(i)}(r) R_{t}^{(0)}(r) \\
& \int \mathrm{d} \Omega \psi^{\left(i^{\prime}\right) *}(\boldsymbol{r}) \mathrm{V}(\boldsymbol{r}) \psi^{(i)}(\boldsymbol{r})=\delta_{i i^{\prime}} \sum_{k} R_{k}^{(i) *}(r) T^{(i)} R_{k}^{(i)}(r)
\end{aligned}
$$

so that the multichannel Schrödinger equation reduces to a coupled system of radial equations for the sets of channels $\left\{u_{t}^{(0)}(r) \equiv r R_{t}^{(0)}(r)\right\}$ and $\left\{u_{k}^{(i)}(r) \equiv r R_{k}^{(i)}(r)\right\}$.

For example, if we considered for simplicity the case of the $b \bar{b}$ component with only one pair, $\left(l_{1}^{(0)}, s_{1}^{(0)}\right)$, coupling to the given $J^{\mathrm{PC}}$, and one meson-meson component $M_{1}^{(1)} \bar{M}_{2}^{(1)}$ with only one pair, $\left(l_{1}^{(1)}, s_{1}^{(1)}\right)$, coupling to the given $J^{\mathrm{PC}}$, the system would read

$$
\left(\begin{array}{cc}
-\frac{1}{2 \mu_{b \bar{b}}}\left(\partial_{r}^{2}-\frac{l_{1}^{(0)}\left(l_{1}^{(0)}+1\right)}{r^{2}}\right)+V_{\mathrm{C}}(r)-E & V_{\text {mix }}^{(1)}(r) \\
V_{\text {mix }}^{(1)}(r) & -\frac{1}{2 \mu^{(1)}}\left(\partial_{r}^{2}-\frac{l_{1}^{(1)}\left(l_{1}^{(1)}+1\right)}{r^{2}}\right)+T^{(1)}-E
\end{array}\right)\left(\begin{array}{c}
u_{1}^{(0)} \\
u_{1}^{(1)}
\end{array}\right)=0
$$

The generalization to any number of possible $\left(l^{(0)}, s^{(0)}\right)$ and $\left(l^{(i)}, s^{(i)}\right), i=1,2 \ldots$ pairs is straightforward by considering each $u_{t}^{(0)}$ and each $u_{k}^{(i)}$ as a component of the eigenfunction. Then, for normalizable solutions of the general system of radial equations, the probability for the $b \bar{b}$ component can be calculated as

$$
\mathcal{P}(b \bar{b})=\sum_{t} \int \mathrm{d} r\left|u_{t}^{(0)}(r)\right|^{2}
$$


and for the $M_{1}^{(i)} \bar{M}_{2}^{(i)}$ component

$$
\mathcal{P}\left(M_{1}^{(i)} \bar{M}_{2}^{(i)}\right)=\sum_{k} \int \mathrm{d} r\left|u_{k}^{(i)}(r)\right|^{2}
$$

Notice that although no direct interaction potential between different meson-meson components is considered, what it is justified for isolated, well separated meson-meson thresholds with no overlap at all, an indirect interaction through their coupling to the $b \bar{b}$ channel is present.

\section{A. Diabatic potential matrix}

The explicit form of the matrix elements $V_{\mathrm{C}}(r), T^{(i)}$, $V_{\text {mix }}^{(i)}(r)$ can be derived from the light field static energies calculated in lattice QCD [8]. As lattice results depend on the chosen lattice spacing the philosophy underlying this derivation is the use of parametrizations motivated from lattice results with parameters to be fixed from phenomenology. Thus, the diagonal element $V_{\mathrm{C}}(r)$ corresponding to the $b-\bar{b}$ potential is parametrized from quenched lattice data on the static quark-antiquark energy [3] as the Cornell potential

$$
V_{\mathrm{C}}(r)=\sigma r-\frac{\chi}{r}-\beta+m_{b}+m_{\bar{b}}
$$

with $\sigma, \chi, \beta$, and $m_{b}$ being the string tension, the color coulomb strength, a constant, and the bottom quark mass, respectively. We shall assume that all the flavor dependence in $V_{\mathrm{C}}(r)$ comes from the mass term. Therefore, we shall keep for hidden-bottom mesons the same values for $\sigma, \chi$, and $\beta$ used in [8] for hidden-charm mesons. In order to fix $m_{b}$ we have to take into account that the potential is spin independent so that the calculated masses should be compared with the experimental mass centroids obtained from spin singlet and spin triplet data. So we choose to fit the $1 P$ ground state mass centroid under the assumption that $1 P_{J}$ experimental resonances are pure bottomonium states, as will be confirmed later on (alternatively we could have chosen to fit the $1 S$ or $2 S$ or $2 P$ mass centroid without any significant change in the forthcoming analysis). Thus, we have

$$
\begin{aligned}
\sigma & =925.6 \mathrm{MeV} / \mathrm{fm}, \\
\chi & =102.6 \mathrm{MeV} \mathrm{fm}, \\
\beta & =855 \mathrm{MeV} . \\
m_{b} & =5215 \mathrm{MeV} .
\end{aligned}
$$

The $b \bar{b}$ spectrum from this Cornell potential for $J^{++}$and $1^{--}$isoscalar states is shown in Table I.

Let us point out that in phenomenological applications of the Cornell potential $[21,22]$ the chosen value of the bottom quark mass differs slightly from ours. In these applications
TABLE I. Bottomonium spectrum from the Cornell potential. Each spectral state is characterized by $J^{\mathrm{PC}}$ and $n L$ quantum numbers. For $J^{\mathrm{PC}}=(0,1,2)^{++}$, it is intended that $F$-wave bottotmonium states appear only for $2^{++}$. Available experimental centroid masses from [20] are listed for comparison.

\begin{tabular}{lccc}
\hline \hline$J^{\text {PC }}$ & $n L$ & Mass $(\mathrm{MeV})$ & Centroid $(\mathrm{MeV})$ \\
\hline$(0,1,2)^{++}$ & $1 P$ & 9900.7 & 9899.7 \\
& $2 P$ & 10254.4 & 10260.2 \\
$1 F$ & 10341.5 & \\
$3 P$ & 10536.6 & \\
$2 F$ & 10601.0 & \\
$4 P$ & 10782.2 & \\
& $1 S$ & 9401.2 & \\
$2 S$ & 9993.8 & \\
$1^{--}$ & $1 D$ & 10150.4 & \\
$3 S$ & 10338.6 & \\
$2 D$ & 10442.0 & \\
$4 S$ & 10615.0 & \\
$3 D$ & 10694.1 & \\
$5 S$ & 10856.4 & \\
\hline \hline
\end{tabular}

distinct values of $\beta$ are considered for bottomonium and charmonium in order to fit approximately the low-lying mass centroids.

Any of the other diagonal elements $T^{(i)}$ represents a $M_{1}^{(i)}-\bar{M}_{2}^{(i)}$ potential. Up to one pion exchange effects that we do not consider this potential is given by the $i$ th mesonmeson threshold

$$
T^{(i)}=m_{M_{1}^{(i)}}+m_{\bar{M}_{2}^{(i)}}
$$

with $m_{M_{1}^{(i)}}$ and $m_{\bar{M}_{2}^{(i)}}$ being the masses of the corresponding mesons. The meson-meson thresholds, calculated from the masses of bottom mesons in [20], are listed in Table II.

It is worth remarking that the use of the experimental masses for the thresholds introduces some implicit spin dependence in the description.

Let us note that each of the $B \bar{B}, B \bar{B}^{*}$, and $B^{*} \bar{B}^{*}$ thresholds is composed of two (approximately) degenerate thresholds. For example $B \bar{B}$ corresponds to $B^{+} B^{-}$and $B^{0} \bar{B}^{0}$, with an experimental threshold mass difference of

TABLE II. Low-lying open-bottom meson-meson thresholds $M_{1}^{(i)} \bar{M}_{2}^{(i)}$. Threshold masses $T^{(i)}$ from the bottom and bottom strange meson masses quoted in [20]. Crossing radii of these thresholds with the Cornell potential, $r_{c}^{(i)}$, are also tabulated.

\begin{tabular}{cccc}
\hline \hline$i$ & $M_{1}^{(i)} \bar{M}_{2}^{(i)}$ & $T^{(i)}(\mathrm{MeV})$ & $r_{c}^{(i)}(\mathrm{fm})$ \\
\hline 1 & $B \bar{B}$ & 10559 & 1.16 \\
2 & $B \bar{B}^{*}$ & 10604 & 1.20 \\
3 & $B^{*} \bar{B}^{*}$ & 10649 & 1.25 \\
4 & $B_{s} \bar{B}_{s}$ & 10733 & 1.33 \\
5 & $B_{s} \bar{B}_{s}^{*}$ & 10782 & 1.38 \\
6 & $B_{s}^{*} \bar{B}_{s}^{*}$ & 10830 & 1.43 \\
\hline \hline
\end{tabular}


$0.6 \mathrm{MeV}$. In contrast the hidden strange cases $B_{s} \bar{B}_{s}, B_{s} \bar{B}_{s}^{*}$, and $B_{s}^{*} \bar{B}_{s}^{*}$ are single thresholds.

The off-diagonal elements, $V_{\text {mix }}^{(i)}(r)$, correspond to $b \bar{b}-M_{1}^{(i)} \bar{M}_{2}^{(i)}$ mixing potentials. From unquenched lattice static energies, calculated for $b \bar{b}$ in the presence of mesonmeson configurations $[4,6]$, the following parametrization has been proposed [8]

$$
\left|V_{\text {mix }}^{(i)}(r)\right|=\frac{\Delta^{(i)}}{2} \exp \left\{-\frac{\left(V_{\mathrm{C}}(r)-T^{(i)}\right)^{2}}{2 \sigma^{2} \rho^{2}}\right\}
$$

where $\rho$ is a radial scale for the mixing, that we shall take equal for all thresholds, and $\Delta^{(i)}$ is a strength parameter corresponding to the difference between the unquenched lattice static energies resulting from the avoided crossing of $V_{\mathrm{C}}(r)$ and $T^{(i)}$ at the crossing radius $r_{c}^{(i)}$ defined by

$$
V_{\mathrm{C}}\left(r_{c}^{(i)}\right)=T^{(i)}
$$

The values of the crossing radii have been tabulated in Table II.

For the sake of simplicity, in $[8,14]$ the same value for $\Delta^{(i)}$ was used for degenerate and single thresholds. Here we go a step further. As shown in the Appendix a doubly degenerate threshold can be managed as an effective single threshold with a different value of $\Delta$ :

$$
\Delta_{\text {degenerate }}=\sqrt{2} \Delta_{\text {single }} .
$$

To make all this clear let us consider for example a system containing $b \bar{b}$ and $B \bar{B}$. From Table II $r_{c}^{(B \bar{B})}=1.16 \mathrm{fm}$. In the lattice calculation of Ref. [4], $r_{c \text { (lattice) }}^{(B \bar{B})}=1.25 \mathrm{fm}$ and $\Delta_{(\text {lattice })}^{(B \bar{B})}$ is close to $50 \mathrm{MeV}$. Hence, we may expect quite a similar value for $\Delta^{(B \bar{B})}$. As for $\rho$ we compare the mixing angle between the ground and excited light field configurations associated to $b \bar{b}$ and $B \bar{B}[8]$ :

$$
\theta(r)=\frac{1}{2} \arctan \left(\frac{2 V_{\text {mix }}^{(B \bar{B})}(r)}{T^{(B \bar{B})}-V_{\mathrm{C}}(r)}\right)
$$

to the one extracted from lattice, see Fig. 15 in [4]. More concretely, by using

$$
\begin{aligned}
\Delta^{(B \bar{B})} & =55 \mathrm{MeV} \\
\rho & =0.3 \mathrm{fm}
\end{aligned}
$$

we obtain the angle and mixing potential drawn in Figs. 1 and 2 respectively.

It is worth remarking that the mixing is only effective in an interval around $r_{c}^{(B \bar{B})}$ determined by the value of $\rho$ and that the sign of the mixing potential has no effect on the results that follow.

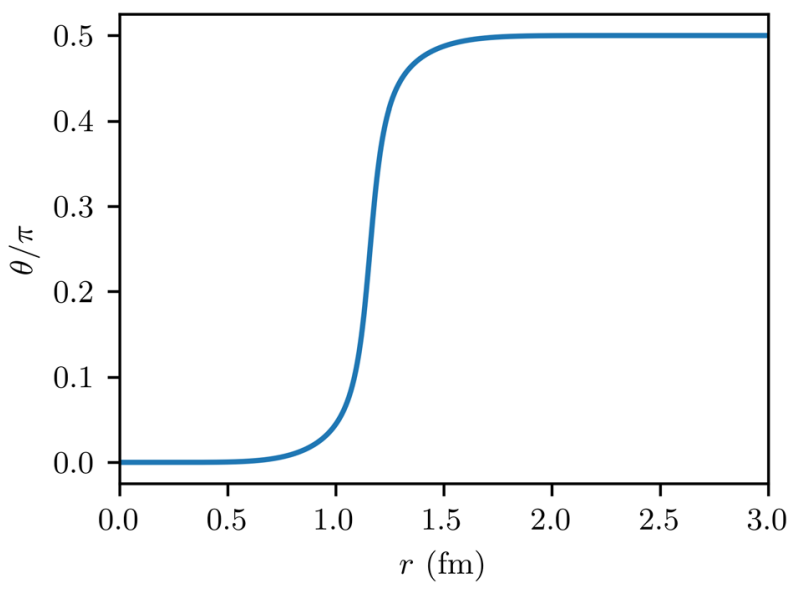

FIG. 1. Mixing angle between $b \bar{b}$ and $B \bar{B}$.

The diabatic potential matrix reads

$$
\left(\begin{array}{cc}
V_{\mathrm{C}}(r) & V_{\text {mix }}^{(B \bar{B})}(r) \\
V_{\text {mix }}^{(B \bar{B})}(r) & m_{B}+m_{\bar{B}}
\end{array}\right)
$$

and its eigenvalues, given by

$$
\begin{aligned}
V_{ \pm}(r) & =\frac{V_{\mathrm{C}}(r)+\left(m_{B}+m_{\bar{B}}\right)}{2} \\
& \pm \sqrt{\left(\frac{V_{\mathrm{C}}(r)-\left(m_{B}+m_{\bar{B}}\right)}{2}\right)^{2}+\left(V_{\text {mix }}^{(B \bar{B})}(r)\right)^{2}}
\end{aligned}
$$

and represented in Fig. 3, should be compared to the static energies for $b \bar{b}$ in the presence of $B \bar{B}$ calculated in lattice, see Figs. 13 and 14 in [4]. (Let us realize that the comparison has to be more qualitative than quantitative since the values of the parameters in the lattice depend on the chosen lattice spacing.)

The extension to a system containing $b \bar{b}, B \bar{B}$, and $B_{s} \bar{B}_{s}$ is straightforward. The diabatic potential matrix is now

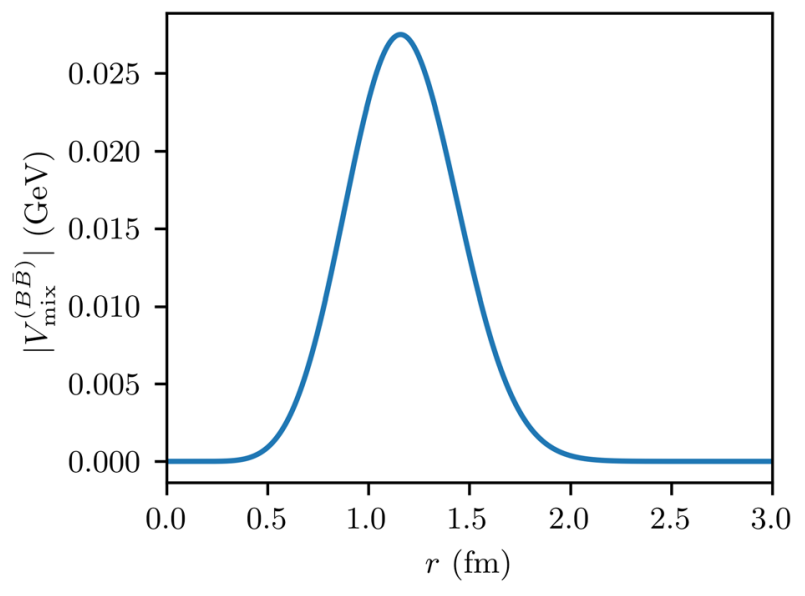

FIG. 2. Mixing potential between $b \bar{b}$ and $B \bar{B}$. 


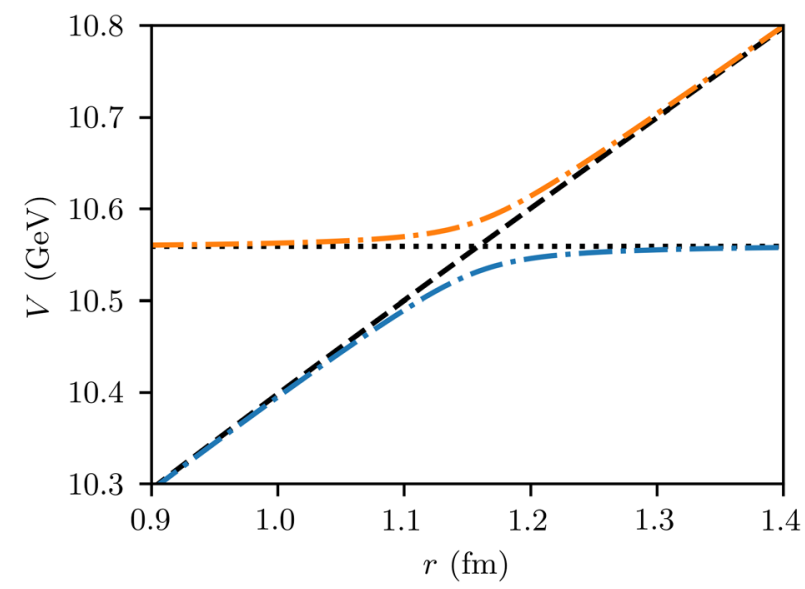

FIG. 3. Static energies. Dashed line: $b \bar{b}$ (Cornell). Dotted line: $B \bar{B}$ threshold. Dash-dotted lines: $r$-dependent eigenvalues of the diabatic potential matrix (20).

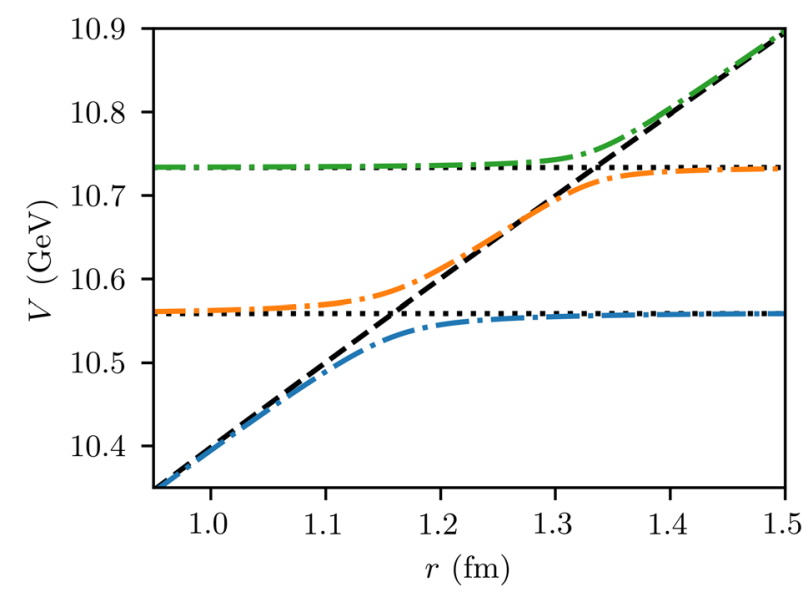

FIG. 4. Static energies. Dashed line: $b \bar{b}$ (Cornell). Dotted lines: $B \bar{B}$ and $B_{s} \bar{B}_{s}$ thresholds. Dash-dotted lines: $r$-dependent eigenvalues of the diabatic potential matrix (22).

$$
\left(\begin{array}{ccc}
V_{\mathrm{C}}(r) & V_{\operatorname{mix}}^{(B \bar{B})}(r) & V_{\operatorname{mix}}^{\left(B_{s} \bar{B}_{s}\right)}(r) \\
V_{\operatorname{mix}}^{(B \bar{B})}(r) & m_{B}+m_{\bar{B}} & 0 \\
V_{\operatorname{mix}}^{\left(B_{s} \bar{B}_{s}\right)}(r) & 0 & m_{B_{s}}+m_{\bar{B}_{s}} \cdot
\end{array}\right)
$$

By using

$$
\Delta^{\left(B_{s} \bar{B}_{s}\right)}=\frac{55}{\sqrt{2}} \mathrm{MeV}
$$

the resulting eigenvalues, plotted in Fig. 4, should be compared to the educated guess of the static energies for $b \bar{b}$ in the presence of $B \bar{B}$ and $B_{s} \bar{B}_{s}$ done in [4] (Fig. 22) and to the lattice calculation performed in [6].

\section{STATE DESCRIPTION}

Any hidden-bottom meson state, characterized by the quantum numbers $J^{\mathrm{PC}}$, with mass below all possible openbottom meson-meson thresholds with the same quantum numbers is stable under decay into open-bottom mesonmeson channels. Hence it corresponds to a bound state solution of the diabatic multichannel Schrödinger equation. On the other hand any $J^{\mathrm{PC}}$ hidden-bottom meson state with mass above a possible open-bottom meson-meson threshold with the same quantum numbers is unstable under decay into open-bottom meson-meson channels and corresponds to a scattering solution of the diabatic multichannel Schrödinger equation.

From a technical point of view the extraction of the values of physical observables from a (normalizable) bound state wave function is straightforward. In contrast, for a scattering wave function it requires the development of a dedicated formalism [23]. Taking into account that the difference in the wave functions is associated to the presence of open meson-meson components in the scattering case, which are asymptotically free, one can approach a scattering resonance solution through a two-step procedure. In the first step one solves a bound state problem incorporating only the closed meson-meson components; in the second step, one generates a resonance through the coupling of the bound state solution to the open meson-meson components. This coupling allows for the calculation of the mass of the resonance through mass corrections to the bound state mass, and for the evaluation of its width. Notice that this procedure is completely nonperturbative. One should keep in mind though the lack of consistency of this bound state based approximation when there is some open threshold giving rise to a mass correction to the bound state that makes the threshold to close with respect to the resulting resonance. This is for example the case for the hidden-charm meson $\psi(4040)$, see [14]. Then, only a direct scattering solution of the multichannel Schrödinger equation can provide a trustable description.

\section{A. Bound states}

In order to calculate bound states a finite number of closed meson-meson thresholds is considered. This is justified because in general for a given bound state the probability of meson-meson components corresponding to thresholds far above the mass of the bound state is expected to be negligible. From our mixing potential, we can estimate that this is the case for any threshold being at least $200 \mathrm{MeV}$ above the mass of the bound state.

The technical procedure to calculate bound states has been detailed elsewhere, see Sec. IV F and Appendices C and $\mathrm{D}$ in [8]. Let us only recall here that in order to avoid possible multiple countings of the same bound state when different sets of closed meson-meson thresholds are considered we assume a one-to-one correspondence with the 
TABLE III. Possible values of $l_{b \bar{b}} \quad\left(s_{b \bar{b}}=1\right)$ and $\left(l_{M_{1}^{(i)} \bar{M}_{2}^{(i)}}, s_{M^{(i)} \bar{M}_{2}^{(i)}}\right)$ for given values of $J^{\mathrm{PC}}$. A missing entry means that the particular meson-meson configuration cannot form a state with the corresponding quantum numbers.

\begin{tabular}{ccccc}
\hline \hline$J^{\mathrm{PC}}$ & $b \bar{b}$ & $B_{(s)} \bar{B}_{(s)}$ & $B_{(s)} \bar{B}_{(s)}^{*}$ & $B_{(s)}^{*} \bar{B}_{(s)}^{*}$ \\
\hline $0^{++}$ & 1 & $(0,0)$ & & $(0,0),(2,2)$ \\
$1^{++}$ & 1 & & $(0,1),(2,1)$ & $(2,2)$ \\
$2^{++}$ & 1,3 & $(2,0)$ & $(2,1)$ & $(0,2),(2,0),(2,2),(4,2)$ \\
$1^{--}$ & 0,2 & $(1,0)$ & $(1,1)$ & $(1,0),(1,2),(3,2)$ \\
\hline \hline
\end{tabular}

bound states of $b \bar{b}$ from the Cornell potential. Hence, each $b \bar{b}$ bound state is the seed of only one bound state, the one obtained when the chosen set of closed thresholds is maximal in the sense of containing the maximum possible number of them. This assumption has proved to work for hidden-charm mesons [8,14], and we shall show it also does for hidden-bottom ones.

Henceforth we center on spin-triplet hidden-bottom mesons with $I=0$ and $J^{\mathrm{PC}}=(0,1,2)^{++}$and $1^{--}$for which there are spectral data available up to $11.0 \mathrm{GeV}$. We restrict our study to bound states with masses up to about $10.8 \mathrm{GeV}$, two hundred $\mathrm{MeV}$ below the first $1^{--} S$-wave meson-meson thresholds. There are several reasons for this. First, it is known the $1^{1} P_{1}$ state $B_{1}(5721)$ but not the corresponding $1^{3} P_{1}$ state $B_{1}($ ?) with an expected similar mass. Hence, the threshold $B_{1} \bar{B}$ is only partially known. Second, $B_{1}(5721)$ has a non-negligible width, $27.5 \pm 3.4 \mathrm{MeV}$, and $B_{1}($ ?) is presumably a much wider state (actually, this may be preventing its experimental detection). Hence, threshold width effects should be properly incorporated. Third, the lowest lying bottomonium hybrid $b \bar{b} g$ ( $g$ : gluon), which could mix with $b \bar{b}$, is predicted to have a mass about $10.9 \mathrm{GeV}$, see [24] and references therein.

The possible values of $l_{b \bar{b}} \quad\left(s_{b \bar{b}}=1\right)$ and $\left(l_{M_{1}^{(i)} \bar{M}_{2}^{(i)}}, s_{M_{1}^{(i)} \bar{M}_{2}^{(i)}}\right)$ coupling to a given $J^{\mathrm{PC}}$ are listed in Table III, where the common notation $B_{(s)}$ to refer to bottom and bottom, strange mesons, and the shorthand $B_{(s)} \bar{B}_{(s)}^{*}$ to denote the $C$-parity eigenstate, are used.

The calculated spectrum of bound states is shown in Table IV.

A glance at the table and its comparison with Table I makes clear that (i) all bound states have a dominant $b \bar{b}$ component, with more than $90 \%$ probability in most cases, (ii) closed meson-meson thresholds give rise to attraction, (iii) the attractive effect on the mass is quantitatively modest, with mass reductions of $16 \mathrm{MeV}$ or less with respect to the $b \bar{b}$ masses obtained from the Cornell potential. These results are in line with the reasonable mass description of known experimental resonances provided by the Cornell potential model.

For a detailed comparison to data we have to take into account that our Cornell potential does not contain spindependent terms. Then, for pure $(n l) b \bar{b}$ states the calculated masses have to be compared to the $(n l)$ experimental centroids; in the other cases, where mesonmeson components are present, since they are specific for any set of $J^{\mathrm{PC}}$ quantum numbers, the comparison has to be done with the experimental candidates with the same $J^{\mathrm{PC}}$. Taking this into consideration all known $J^{\mathrm{PC}}=(0,1,2)^{++}$ and $1^{--}$experimental resonances below $10.8 \mathrm{GeV}$ can be assigned to bound states with the same location (below or above) with respect to the meson-meson thresholds. Thus, we see that the calculated mass for the $(0,1,2)^{++}$ground states, which are $100 \%$ Cornell $(1 P) b \bar{b}$ states, coincides with the experimental mass centroid from $1 P_{J}$ states at $9899.9 \pm 0.6 \mathrm{MeV}$. Actually, this coincidence has been required to fix the bottom quark mass. As for the first excited $(0,1,2)^{++}$states, which are $100 \%$ Cornell $(2 P) b \bar{b}$ states, the calculated mass is very close to the experimental mass centroid from $2 P_{J}$ states at $10260.2 \pm 0.7 \mathrm{MeV}$. The only additional $\mathrm{J}^{++}$pure Cornell state is the second excitation of $2^{++}$, assigned to the $\left(1 F_{2}\right) b \bar{b}$ state with a predicted mass of about $10340 \mathrm{MeV}$.

The second excited $(0,1)^{++}$and the third excited $2^{++}$ states are predicted to contain more than a $90 \%$ of $(3 P) b \bar{b}$ and less than a $10 \%$ of meson-meson components. For $1^{++}$ and $2^{++}$the calculated masses compare well with existing data (for $0^{++}$there is no PDG data). Indeed the measured masses of $\chi_{b 1}(3 P), \quad 10513.42 \pm 0.41 \pm 0.53 \mathrm{MeV}$, and $\chi_{b 2}(3 P), \quad 10524.02 \pm 0.57 \pm 0.53 \mathrm{MeV}$, differ from the calculated values by less than $30 \mathrm{MeV}$. It is worth mentioning that the presence of meson-meson components makes the calculated masses to be $12 \mathrm{MeV}$ closer to data than the corresponding Cornell masses suggesting that a renaming of these resonances as $\chi_{b 1}(10513)$ and $\chi_{b 2}(10524)$ might be in order.

From the point of view of its meson-meson composition the most interesting $\mathrm{J}^{++}$case is the third excited state of $1^{++}$with a significant $24 \%$ of $B_{s} \bar{B}_{s}^{*}$. This significant percentage has to do with the immediate vicinity of the Cornell $(4 P) b \bar{b}$ state and the $B_{s} \bar{B}_{s}^{*}$ threshold, both located at about $10782.2 \mathrm{MeV}$. Notice though that the mass shift due to $B_{s} \bar{B}_{s}^{*}$ is only $7 \mathrm{MeV}$ with respect to the Cornell $(4 P)$ $b \bar{b}$ mass. More importantly, as $B_{s} \bar{B}_{s}^{*}$ can naturally decay strongly into $\Upsilon(1 S) \phi$ through quark exchange this could be a possible discovery channel.

For $1^{--}$the ground and the first three excited states are predicted to be $100 \%$ the Cornell $(1 S, 2 S, 1 D, 3 S) b \bar{b}$ states respectively. For the ground state $(1 S)$ the difference between the experimental centroid (from $\eta_{b}(1 S)$ and $\Upsilon(1 S))$ at $9445.0 \pm 0.7 \mathrm{MeV}$ and the calculated mass is $45 \mathrm{MeV}$, significantly higher than in any other case. This could be indicating the presence of more relevant relativistic effects in the $1 S$ state. Indeed, for the first excited state $(2 S)$ the difference between the $\left(\eta_{b}(2 S), \Upsilon(2 S)\right)$ mass centroid at $10017.20 \pm 1.8 \mathrm{MeV}$ and the calculated value gets reduced to $23 \mathrm{MeV}$. For the $(1 D)$ and $(3 S)$ states the lack of data prevents the evaluation of the mass centroids 
TABLE IV. Calculated masses, $b \bar{b}$ and meson-meson component probabilities, for $J^{\mathrm{PC}}$ bottomoniumlike bound state solutions. Vanishing and negligible (i.e., inferior to $1 \%$ ) probabilities are not displayed.

\begin{tabular}{|c|c|c|c|c|c|c|c|c|}
\hline$J^{\mathrm{PC}}$ & Mass $(\mathrm{MeV})$ & $b \bar{b}$ & $B \bar{B}$ & $B \bar{B}^{*}$ & $B^{*} \bar{B}^{*}$ & $B_{s} \bar{B}_{s}$ & $B_{s} \bar{B}_{s}^{*}$ & $B_{s}^{*} \bar{B}_{s}^{*}$ \\
\hline \multirow[t]{4}{*}{$0^{++}$} & 9900.7 & $100 \%$ & & & & & & \\
\hline & 10254.1 & $100 \%$ & & & & & & \\
\hline & 10530.2 & $91 \%$ & $8 \%$ & & $1 \%$ & & & \\
\hline & 10778.1 & $98 \%$ & & & & & & $2 \%$ \\
\hline \multirow[t]{4}{*}{$1^{++}$} & 9900.7 & $100 \%$ & & & & & & \\
\hline & 10254.2 & $100 \%$ & & & & & & \\
\hline & 10532.1 & $97 \%$ & & $3 \%$ & & & & \\
\hline & 10775.1 & $75 \%$ & & & & & $24 \%$ & $1 \%$ \\
\hline \multirow[t]{6}{*}{$2^{++}$} & 9900.7 & $(100,0) \%$ & & & & & & \\
\hline & 10253.9 & $(100,0) \%$ & & & & & & \\
\hline & 10340.9 & $(0,100) \%$ & & & & & & \\
\hline & 10527.7 & $(92,2) \%$ & $3 \%$ & $1 \%$ & $2 \%$ & & & \\
\hline & 10592.7 & $(2,91) \%$ & & $3 \%$ & $4 \%$ & & & \\
\hline & 10776.2 & $(91,1) \%$ & & & & & $4 \%$ & $4 \%$ \\
\hline \multirow[t]{7}{*}{$1^{--}$} & 9401.2 & $(100,0) \%$ & & & & & & \\
\hline & 9993.8 & $(100,0) \%$ & & & & & & \\
\hline & 10150.3 & $(0,100) \%$ & & & & & & \\
\hline & 10337.2 & $(100,0) \%$ & & & & & & \\
\hline & 10439.4 & $(0,99) \%$ & $1 \%$ & & & & & \\
\hline & 10598.8 & $(70,3) \%$ & & $21 \%$ & $6 \%$ & & & \\
\hline & 10691.3 & $(0,98) \%$ & & & & $1 \%$ & & $1 \%$ \\
\hline
\end{tabular}

for comparison. Instead, we can check that the calculated mass for $(1 D)$ is pretty close to the measured mass of $\Upsilon_{2}(1 D), 10163.7 \pm 1.4 \mathrm{MeV}$, and that the calculated mass of $(3 S)$ is lower than the measured mass of $\Upsilon(3 S)$, $10355.2 \pm 0.5 \mathrm{MeV}$, as should be expected.

All the higher excited states contain meson-meson components. However, only for the fifth excited state, which contains a dominant $(70 \%)$ Cornell $(4 S) b \bar{b}$ component, we predict a significant meson-meson probability ( $21 \%$ of $\left.B \bar{B}^{*}\right)$, due to the vicinity of the $(4 S) b \bar{b}$ state and the $B \bar{B}^{*}$ threshold. This suggests that for the corresponding

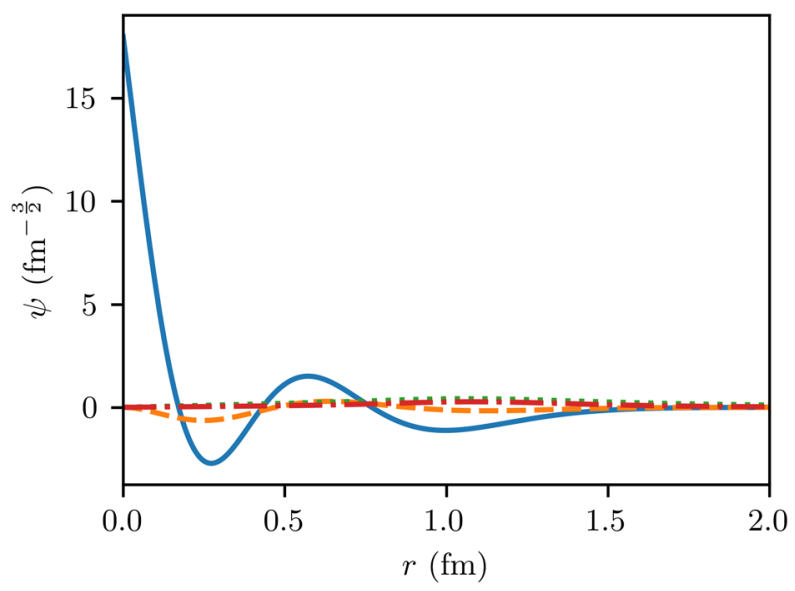

FIG. 5. Radial wave function of the fifth excited $1^{--}$state. $b \bar{b}(4 S), b \bar{b}(3 D), B \bar{B}^{*}\left(l_{B \bar{B}^{*}}=1\right)$, and $B^{*} \bar{B}^{*}\left(l_{B^{*} \bar{B}^{*}}=1\right)$ components are drawn with a solid, dashed, dotted, and dash-dotted line respectively. experimental resonance the label $\Upsilon(10580)$ should be preferred to the PDG alternative $\Upsilon(4 S)$.

It is illustrative to plot the radial wave function of this state for the several components, see Fig. 5.

We see that the presence of meson-meson components makes the radial wave function to extend to larger distance than the Cornell $(4 S) b \bar{b}$ one, and correlated with this there is a loss of probability density at the origin $(r=0)$ as compared to the Cornell case. This could explain the discrepancies observed between the calculated leptonic width ratios in the Cornell model and data. More concretely, the $1^{--}$leptonic width ratios are calculated from [25]

$$
\frac{\Gamma\left(\Upsilon^{\left(n_{1}\right)} \rightarrow e^{+} e^{-}\right)}{\Gamma\left(\Upsilon^{\left(n_{2}\right)} \rightarrow e^{+} e^{-}\right)}=\left|\frac{R_{\Upsilon^{\left(n_{1}\right)}}(0)}{R_{\Upsilon^{\left(n_{2}\right)}}(0)}\right|^{2} \frac{m_{\Upsilon^{\left(n_{2}\right)}}^{2}}{m_{\Upsilon^{\left(n_{1}\right)}}^{2}}
$$

where $R_{\Upsilon^{(n)}}(0)$ stands for the radial wave function at the origin and $m_{\Upsilon^{(n)}}$ for the mass of the $\Upsilon$ state containing a $(n S) b \bar{b}$ component. The calculated values for these ratios and their comparison to data are given in Table V (the use of the experimental masses instead of the calculated ones would not make any difference).

A look at the table makes clear that (i) the ratios involving $\Gamma\left(\Upsilon(1 S) \rightarrow e^{+} e^{-}\right)$, are deficiently described by both the Cornell model (with the exception of $\left.\frac{\Gamma\left(\Upsilon(10580) \rightarrow e^{+} e^{-}\right)}{\Gamma\left(\Upsilon(1 s) \rightarrow e^{+} e^{-}\right)}\right)$and the diabatic approach, (ii) the diabatic values for these ratios can be put in accord with data through multiplication by a common factor of $\simeq 1.3$, (iii) all 
TABLE V. Calculated leptonic width ratios from the Cornell model and the diabatic approach, as compared to data from [20].

\begin{tabular}{lccc}
\hline \hline Leptonic Width Ratio & Cornell & Experiment & Diabatic \\
\hline$\frac{\Gamma\left(\Upsilon(2 s) \rightarrow e^{+} e^{-}\right)}{\Gamma\left(\Upsilon(1 s) \rightarrow e^{+} e^{-}\right)}$ & 0.36 & $0.456 \pm 0.14$ & 0.36 \\
$\frac{\Gamma\left(\Upsilon(3 s) \rightarrow e^{+} e^{-}\right)}{\left.\Gamma((1) s) \rightarrow e^{e} e^{-}\right)}$ & 0.25 & $0.33 \pm 0.1$ & 0.25 \\
$\frac{\Gamma\left(\Upsilon(10580) \rightarrow e^{+} e^{-}\right)}{\Gamma\left(\Upsilon(1 s) \rightarrow e^{+} e^{-}\right)}$ & 0.21 & $0.20 \pm 0.02$ & 0.14 \\
$\frac{\Gamma\left(\Upsilon(3 s) \rightarrow e^{+}+e^{-}\right)}{\left.\Gamma((2) s) \rightarrow e^{+} e^{-}\right)}$ & 0.70 & $0.72 \pm 0.03$ & 0.70 \\
$\frac{\Gamma\left(\Upsilon(1058) \rightarrow e^{+} e^{-}\right)}{\Gamma\left(\Upsilon(2 s) \rightarrow e^{+} e^{-}\right)}$ & 0.58 & $0.44 \pm 0.06$ & 0.39 \\
$\frac{\Gamma\left((10580) \rightarrow e^{+} e^{-}\right)}{\Gamma\left(\Upsilon(3 s) \rightarrow e^{+} e^{-}\right)}$ & 0.82 & $0.61 \pm 0.08$ & 0.56 \\
\hline \hline
\end{tabular}

the ratios not involving $\Gamma\left(\Upsilon(1 S) \rightarrow e^{+} e^{-}\right)$are well described by the diabatic approach whereas the Cornell model is far from data except $\frac{\Gamma\left(\Upsilon(3 s) \rightarrow e^{+} e^{-}\right)}{\Gamma\left(\Upsilon(2 s) \rightarrow e^{+} e^{-}\right)}$for which there is no difference in the calculated wave functions with both approximations.

These results suggest that the failure of the diabatic approach regarding the ratios involving $\Gamma\left(\Upsilon(1 S) \rightarrow e^{+} e^{-}\right)$ may have to do with the presence of relativistic corrections in $\Upsilon(1 S)$ making its radial wave function at the origin to decrease a $14 \%$.

Therefore, we may tentatively conclude that data from leptonic widths can be taken as an indication of the mesonmeson compositeness of $\Upsilon(10580)$. In this regard, it is also interesting to add that it is the $b \bar{b}(4 S)-B \bar{B}^{*}$ interaction the main physical mechanism underlying the explanation of the leptonic widths. The small $D$ mixing, $3 \%$ of $b \bar{b}(3 D)$, which is mainly induced through the $S$ and $D$ coupling to $B^{*} \bar{B}^{*}$, plays a marginal quantitative role. Moreover, other sources of $S-D$ mixing such as a direct tensor interaction within the Cornell potential, should also have a quite limited importance in order to preserve the accurate leptonic width description. This is in contrast to other explanations in the literature based on a significant $S-D$ mixing, see for instance [26].

The calculated sixth excited state has a predicted mass close to $10700 \mathrm{MeV}$ and it is very dominantly a $b \bar{b}(3 D)$ state. It could be possibly assigned to the not well established $\Upsilon(10753)$ with a measured mass of $10752.7 \pm 5.9_{-1.1}^{+0.7} \mathrm{MeV}$. However, the discovery channel $\Upsilon(n S) \pi^{+} \pi^{-}$with $n=1,2,3$ is not expected to be a dominant channel for a $(3 D)$ state suggesting that some mixing with the $b \bar{b}(4 S)$ state is lost. This can be due to the fact that the $B \bar{B}^{*}$, and particularly the $B^{*} \bar{B}^{*}$ thresholds, which according to our previous discussion can give rise to this mixing, are not taken into account in our bound state approach for the sixth excited state since they are open meson-meson channels. Indeed, we shall show later on that this excitation has a prominent width to $B^{*} \bar{B}^{*}$. Hence, a significant $D-S$ mixing through the coupling to $B^{*} \bar{B}^{*}$ could be present. The theoretical description of this mixing would require a complete (scattering) solution of the problem which is out of the scope of our current analysis.

\section{B. Mass corrections and widths}

Let us realize that for pure Cornell states, with masses below the first meson-meson threshold, there are no open meson-meson channels. Hence, no widths, and mass corrections being mostly limited to spin splittings which are known to be quantitatively important for these states. Indeed, the derivation of the form of the spin dependent potential terms from QCD, and a numerical evaluation of the Cornell spin splittings for $b \bar{b}$ was carried out forty years ago [27]. We simply copy here those results for the slightly different values we use for the parameters of the Cornell potential hardly makes a difference. The corrected masses for the pure Cornell states in Table IV are given in Table VI.

We see that a very good mass description is obtained. The biggest difference between the calculated mass and data is of $35 \mathrm{MeV}$ for $\Upsilon(1 S)$ which we may attribute, at least partially, to further relativistic (kinetic energy) effects.

To proceed to a similar evaluation of spin splitting for states with meson-meson components, spin dependent terms of the mixing and meson-meson potentials should be taken into account as well. However, the complete lack of knowledge of the spin dependence in the mixing potential prevents carrying out this procedure. Instead, for states with mass above the first meson-meson threshold, we can evaluate mass corrections and widths from the open meson-meson thresholds neglected in the bound state calculation. The nonperturbative method we follow for this evaluation has been explained elsewhere, see [14] and references therein. Let us only recall here that the physical effect of the coupling to the continuum is to dilute the bound state through a band of stationary scattering states, giving rise to a resonance. If we call $m_{\mathrm{bs}}$ the mass of the bound state, $M_{1}^{(j)} \bar{M}_{2}^{(j)}$ with $j=1,2 \ldots, n$ the corresponding

TABLE VI. Spin splittings (in MeV) for pure Cornell spin triplet states. The corrected masses (in $\mathrm{MeV}$ ) and their comparison to the measured masses [20] of the assigned mesons are also shown.

\begin{tabular}{lrrcc}
\hline \hline$J^{\mathrm{PC}}(n l)$ & Splitting & \multicolumn{1}{c}{ Mass } & Experiment & Meson \\
\hline $1^{--}(1 S)$ & 23.7 & 9424.9 & $9460.30 \pm 0.26$ & $\Upsilon(1 S)$ \\
$0^{++}(1 P)$ & -35.8 & 9864.9 & $9859.44 \pm 0.42 \pm 0.31$ & $\chi_{b 0}(1 P)$ \\
$1^{++}(1 P)$ & -11.0 & 9889.7 & $9892.78 \pm 0.26 \pm 0.31$ & $\chi_{b 1}(1 P)$ \\
$2^{++}(1 P)$ & 13.8 & 9914.5 & $9912.21 \pm 0.26 \pm 0.31$ & $\chi_{b 2}(1 P)$ \\
$1^{--}(2 S)$ & 10.3 & 10004.1 & $10023.26 \pm 0.31$ & $\Upsilon(2 S)$ \\
$1^{--}(1 D)$ & -3.3 & 10147.0 & $10163.7 \pm 1.4$ & $\Upsilon_{2}(1 D)$ \\
$0^{++}(2 P)$ & -26.4 & 10227.7 & $10232.5 \pm 0.4 \pm 0.5$ & $\chi_{b 0}(2 P)$ \\
$1^{++}(2 P)$ & -8.2 & 10246.0 & $10255.46 \pm 0.22 \pm 0.50$ & $\chi_{b 1}(2 P)$ \\
$2^{++}(2 P)$ & 10.2 & 10264.1 & $10268.65 \pm 0.22 \pm 0.50$ & $\chi_{b 2}(2 P)$ \\
$1^{--}(3 S)$ & 7.8 & 10345.0 & $10355.2 \pm 0.5$ & $\Upsilon(3 S)$ \\
\hline \hline
\end{tabular}


open meson-meson components, and $m_{\text {res }}$ and $\Gamma$ the mass and width respectively of the resulting resonance, then

$$
m_{\mathrm{res}}-m_{\mathrm{bs}}=\sum_{j, k} \mathcal{P} \int \mathrm{d} E^{(j)} \mu^{(j)} \frac{p^{(j)}}{m_{\mathrm{res}}-E^{(j)}}\left|\mathcal{I}_{k}^{(j)}\left(p^{(j)}\right)\right|^{2}
$$

and

$$
\frac{\Gamma}{2}=\sum_{j, k} \pi p^{(j)}\left|\mathcal{I}_{k}^{(j)}\left(p^{(j)}\right)\right|_{E^{(j)}=m_{\mathrm{res}}}^{2}
$$

where $\mathcal{P} \int$ stands for the Cauchy principal value integral, $E^{(j)}$ for the meson-meson energy in the center of mass frame (resonance at rest)

$$
E^{(j)}=E_{M_{1}^{(j)}}+E_{\bar{M}_{2}^{(j)}}
$$

with

$$
\begin{aligned}
& E_{M_{1}^{(j)}}=m_{M_{1}^{(j)}}+\frac{\left(p^{(j)}\right)^{2}}{2 m_{M_{1}^{(j)}}} \\
& E_{\bar{M}_{2}^{(j)}}=m_{\bar{M}_{2}^{(j)}}+\frac{\left(p^{(j)}\right)^{2}}{2 m_{\bar{M}_{2}^{(j)}}}
\end{aligned}
$$

where $p^{(j)}$ is the meson momentum, $\mu^{(j)}$ for the reduced mass of $M_{1}^{(j)}$ and $\bar{M}_{2}^{(j)}$

$$
\mu^{(j)} \equiv \frac{m_{M_{1}^{(j)}} m_{\bar{M}_{2}^{(j)}}}{m_{M_{1}^{(j)}}+m_{\bar{M}_{2}^{(j)}}}
$$

and

$\mathcal{I}_{k}^{(j)}\left(p^{(j)}\right) \equiv \sqrt{\frac{2}{\pi}} i^{-l_{k}^{(j)}} \int \mathrm{d} r r^{2} j_{l_{k}^{(j)}}\left(p^{(j)} r\right) V_{\text {mix }}^{(j)}(r)\left(\sum_{t} R_{t}^{(0)}(r)\right)$

where $j_{l}$ is the spherical Bessel function.

It should be emphasized that our calculation of mass corrections and widths does not introduce any new parameter, so the comparison of the masses and widths of the resulting resonances with data may serve as a stringent test of the mixing interaction.

The calculated masses of the resonances $m_{\text {res }}$, as well as their differences with the masses of the corresponding bound states, $m_{\mathrm{res}}-m_{\mathrm{bs}}$, are shown in Table VII. For the sake of completeness we have also included in the table non pure Cornell bound states whose masses are below the first open-bottom meson-meson threshold and, consequently there is no mass correction.
TABLE VII. Calculated mass corrections, $m_{\text {res }}-m_{\text {bs }}$, and total masses, $m_{\text {res }}$, in $\mathrm{MeV}$, for non pure Cornell $J^{\mathrm{PC}}=(0,1,2)^{++}$and $1^{--}$states below $10.8 \mathrm{GeV}$. Measured masses from [20], when existing, corresponding to the meson type assignment in the last column (the subscript referring to the dominant $b \bar{b}$ component), are also given for comparison.

\begin{tabular}{ccccc}
\hline \hline$J^{\text {PC }}$ & $m_{\text {res }}-m_{\text {bs }}$ & $m_{\text {res }}$ & Experiment & Meson \\
\hline $0^{++}$ & 0 & 10530.2 & & $\left(\chi_{b 0}\right)_{3 P}$ \\
& 7.7 & 10785.8 & & $\left(\chi_{b 0}\right)_{4 P}$ \\
$1^{++}$ & 0 & 10532.1 & $10513.42 \pm 0.41 \pm 0.53$ & $\chi_{b 1}(10513)$ \\
& 3.8 & 10778.9 & & $\left(\chi_{b 1}\right)_{4 P}$ \\
$2^{++}$ & 0 & 10527.7 & $10524.02 \pm 0.57 \pm 0.53$ & $\chi_{b 2}(10524)$ \\
& -4.3 & 10588.4 & & $\left(\chi_{b 2}\right)_{2 F}$ \\
& 6.1 & 10782.3 & & $\left(\chi_{b 2}\right)_{4 P}$ \\
$1^{--}$ & 0 & 10439.4 & & $(\Upsilon)_{2 D}$ \\
& 1.0 & 10599.8 & $10579.4 \pm 1.2$ & $\Upsilon(10580)$ \\
& 5.7 & 10697.0 & $10752.7 \pm 5.9_{-1.1}^{+0.7}$ & $\Upsilon(10753)$ \\
\hline \hline
\end{tabular}

As can be checked the calculated masses are in good agreement (less than $20 \mathrm{MeV}$ mass difference) with the few existing data, except for $\Upsilon(10753)$. This may be indicating that the non considered spin splittings are not quantitatively as important as for pure Cornell states. On the other hand, the mass corrections due to open thresholds are quantitatively small, of a few $(<10) \mathrm{MeV}$ at most. Altogether, these arguments make us confident about the predicted masses for the not yet discovered resonances. Regarding $\Upsilon(10753)$ the deficient predicted mass may be indicating the lack of a significant $D-S$ mixing which could arise from the coupling to $B^{*} \bar{B}^{*}$ as well as from the incorporation of spin dependent terms in the diabatic potential matrix.

As for the decay widths to open-bottom meson-meson channels, we have summarized them in Table VIII.

It is remarkable that the calculated values are in very good agreement with the few existing data. So, for $\Upsilon(10580)$ and $\Upsilon(10753)$ the predicted values of the total widths are fully compatible with data. For $\Upsilon(10580)$ there is only one decay channel $B \bar{B}$ contributing to the width. Experimentally this channel saturates with more than 96\% the total width. For the not well established $\Upsilon(10753)$ we predict a very dominant decay into $B^{*} \bar{B}^{*}$

TABLE VIII. Total masses, $m_{\text {res}}$, and decay widths to openbottom meson-meson channels, in $\mathrm{MeV}$, of bottomoniumlike states above threshold. Available experimental widths from [20] are quoted for comparison.

\begin{tabular}{lrrrrrrr}
\hline \hline$J^{\mathrm{PC}}$ & $m_{\mathrm{res}}$ & $\Gamma_{B \bar{B}}$ & $\Gamma_{B \bar{B}^{*}}$ & $\Gamma_{B^{*} \bar{B}^{*}}$ & $\Gamma_{B_{s} \bar{B}_{s}}$ & $\Gamma_{\text {tot }}^{\mathrm{Theor}}$ & $\Gamma_{\mathrm{tot}}^{\mathrm{Exp}}$ \\
\hline $0^{++}$ & 10785.8 & 1.6 & & 5.3 & 0.7 & 7.6 & \\
$1^{++}$ & 10778.9 & & 0.2 & 1.7 & & 1.9 & \\
$2^{++}$ & 10588.4 & 4.3 & & & & 4.3 & \\
& 10782.3 & 5.4 & 1.5 & 21.0 & 10.4 & 38.3 & \\
$1^{--}$ & 10599.8 & 21.9 & & & & 21.9 & $20.5 \pm 2.5$ \\
& 10697.0 & 2.0 & 1.0 & 38.0 & & 41.0 & $36_{-11.3-3.3}^{+17.6+3.9}$ \\
\hline \hline
\end{tabular}


which could help to guide new experimental searches. It is also worth to emphasize the pretty small values of the widths predicted for some not yet discovered $(0,1,2)^{++}$ resonances, in particular for the third excited $1^{++}$state with a value smaller than $2 \mathrm{MeV}$. Although we should add to these predictions some uncertainty we consider them encouraging for experimental analyses. In the $1^{++}$case, its small width (to $B^{*} \bar{B}^{*}$ and $B^{*} \bar{B}^{*}$ ), and its $B_{s} \bar{B}_{s}^{*}$ content (see Table IV) pointing out to a significant $\Upsilon(1 S) \phi$ decay, can help to its experimental discovery.

It should be mentioned that the effect of meson-meson thresholds in the hidden-bottom spectrum has been previously studied in the literature, see for instance [28-30] and references therein. Quite generally all these studies make use of the ${ }^{3} P_{0}$ quark pair creation mechanism to mix the $b \bar{b}$ and meson-meson components. This ${ }^{3} P_{0}$ model lacks direct justification from QCD. Instead, we use a mixing potential whose form is directly based on lattice results for the static light field energies for $b \bar{b}$ in the presence of meson-meson configurations. This gives rise to an utterly different mixing description which is enhanced around the crossing points of the Cornell potential with the meson-meson thresholds. Moreover, in most of these previous studies a perturbative hadron-loop treatment has been followed to calculate mass corrections and widths from meson-meson thresholds. This is conceptually questionable since the perturbative series is divergent as shown in [14]. Much more related to our work is a quite recent analysis [19] where the authors proceed to a numerical parametrization of the diabatic potential matrix from lattice data and to the evaluation from it of the scattering solutions of the Schrödinger equation. However, due to the formidable difficulty of the problem only $b \bar{b} S$-waves and a reduced number of thresholds masses (at most two) have been considered until now, what reduces considerably its predictive power.

\section{SUMMARY}

We have applied the diabatic approach, which allows for a QCD based analysis of the heavy-quark meson spectra, to the description of $I=0, J^{\mathrm{PC}}=(0,1,2)^{++}$and $1^{--}$hiddenbottom mesons with masses below $10.8 \mathrm{GeV}$. More precisely, we have solved the Schrödinger equation for $b \bar{b}$ and meson-meson components with the same $J^{\mathrm{PC}}$ quantum numbers. The form of the diabatic potential matrix entering in this equation has been derived from current lattice data on the energy of static $b$ and $\bar{b}$ sources, when $b \bar{b}$ mixes with $B \bar{B}$ and $B_{s} \bar{B}_{s}$ configurations. For practical purposes, we have followed a bound state based approximation to describe resonances. From it mass corrections and widths have been properly incorporated. A good description of masses, decay widths to open-bottom meson-meson channels, and (for $1^{--}$) leptonic widths of known resonances has been obtained. Of particular interest is the prediction of a significantly lower than $1 b \bar{b}$ probability in $\Upsilon(10580)$ which allows for an accurate description of its leptonic width ratios with the $\Upsilon((2,3) S)$ states. Concerning the not yet discovered resonances it is noteworthy the prediction of a narrow $\chi_{b 1}(10779)$, possibly with a significant decay into $\Upsilon(1 S) \phi$ as a consequence of its $B_{s} \bar{B}_{s}^{*}$ content.

These results for bottomoniumlike mesons give additional support to the diabatic approach in QCD as an appropriate framework for a unified and complete nonperturbative description of conventional and unconventional heavy-quark mesons. They make also clear that the presence of meson-meson thresholds introduces a flavor spectral dependence so that a flavor independent partner correspondence between the hidden-bottom and the hidden-charm mesons is limited to the conventional bottomonium, pure $b \bar{b}$, and charmonium, pure $c \bar{c}$, states.

\section{ACKNOWLEDGMENTS}

This work has been supported by Ministerio de Economía, Industria y Competitividad of Spain and European Regional Development Fund Grant No. FPA2016-77177-C2-1-P, by EU Horizon 2020 Grant No. 824093 (STRONG-2020), and by Ministerio de Ciencia e Innovación and Agencia Estatal de Investigación of Spain and European Regional Development Fund Grant No. PID2019-105439 GBC21. R. B. acknowledges a FPI fellowship from Ministerio de Ciencia, Innovacíon y Universidades of Spain under Grant No. BES-2017-079860.

\section{APPENDIX: DEGENERATE THRESHOLDS}

Let us consider for instance the (almost) degenerate $B^{+} B^{-}$and $B^{0} \bar{B}^{0}$ thresholds.

In the diabatic formalism the potential matrix for a physical system containing $b \bar{b}, B^{+} B^{-}$, and $B^{0} \bar{B}^{0}$ components reads

$$
\left(\begin{array}{ccc}
V_{\mathrm{C}}(r) & V_{\text {mix }}^{(1)}(r) & V_{\text {mix }}^{(2)}(r) \\
V_{\text {mix }}^{(1)}(r) & T_{B^{+} B^{-}} & 0 \\
V_{\text {mix }}^{(2)}(r) & 0 & T_{B^{0} \bar{B}^{0}}
\end{array}\right)
$$

with

$$
\begin{aligned}
V_{\text {mix }}^{(1)}(r) & =\left\langle\zeta_{b \bar{b}}\left|H_{\text {static }}^{\mathrm{lf}}(\boldsymbol{r})\right| \zeta_{B^{+} B^{-}}\right\rangle \\
V_{\text {mix }}^{(2)}(r) & =\left\langle\zeta_{b \bar{b}}\left|H_{\text {static }}^{\mathrm{lf}}(\boldsymbol{r})\right| \zeta_{B^{0} \bar{B}^{0}}\right\rangle \\
V_{\mathrm{C}}(r) & =\left\langle\zeta_{b \bar{b}}\left|H_{\text {static }}^{\mathrm{lf}}(\boldsymbol{r})\right| \zeta_{b \bar{b}}\right\rangle \\
T_{B^{+} B^{-}} & =\left\langle\zeta_{B^{+} B^{-}}\left|H_{\text {static }}^{\mathrm{lf}}(\boldsymbol{r})\right| \zeta_{B^{+} B^{-}}\right\rangle \\
T_{B^{0} \bar{B}^{0-}} & =\left\langle\zeta_{B^{0} \bar{B}^{0}}\left|H_{\text {static }}^{\mathrm{lf}}(\boldsymbol{r})\right| \zeta_{B^{0} \bar{B}^{0}}\right\rangle
\end{aligned}
$$


where $H_{\text {static }}^{\mathrm{lf}}(\boldsymbol{r})$ denotes the Hamiltonian for the light fields (gluons and light quarks), and $\left|\zeta_{b \bar{b}}\right\rangle,\left|\zeta_{B^{+} B^{-}}\right\rangle,\left|\zeta_{B^{0} \bar{B}^{0}}\right\rangle$, the ground and excited light field configurations associated to the $b \bar{b}, B^{+} B^{-}$, and $B^{0} \bar{B}^{0}$ components respectively [8].

If we neglect the small mass difference between $T_{B^{+} B^{-}}$ and $T_{B^{0} \bar{B}^{0}}$, assume $V_{\text {mix }}^{(1)}(r)=V_{\text {mix }}^{(2)}(r) \equiv V_{\text {mix }}(r)$, and define the light field configurations

$$
\begin{aligned}
& \left|\zeta_{(B \bar{B})_{0}}\right\rangle=\frac{1}{\sqrt{2}}\left|\zeta_{B^{+} B^{-}}\right\rangle-\frac{1}{\sqrt{2}}\left|\zeta_{B^{0} \bar{B}^{0}}\right\rangle \\
& \left|\zeta_{(B \bar{B})_{1}}\right\rangle=\frac{1}{\sqrt{2}}\left|\zeta_{B^{+} B^{-}}\right\rangle+\frac{1}{\sqrt{2}}\left|\zeta_{B^{0} \bar{B}^{0}}\right\rangle
\end{aligned}
$$

then the expression of the diabatic potential matrix in terms of these light field configurations reads

$$
\left(\begin{array}{ccc} 
& & \\
V_{\mathrm{C}}(r) & \sqrt{2} V_{\text {mix }}(r) & 0 \\
\sqrt{2} V_{\text {mix }}(r) & T_{B \bar{B}} & 0 \\
0 & 0 & T_{B \bar{B}}
\end{array}\right)
$$

where $T_{B \bar{B}}=T_{B^{+} B^{-}}=T_{B^{0} \bar{B}^{0}}$ and

$$
\begin{aligned}
& \left\langle\zeta_{b \bar{b}}\left|H_{\text {static }}^{\mathrm{lf}}(\boldsymbol{r})\right| \zeta_{(B \bar{B})_{0}}\right\rangle=\sqrt{2} V_{\text {mix }}(r) \\
& \left\langle\zeta_{b \bar{b}}\left|H_{\text {static }}^{\mathrm{lf}}(\boldsymbol{r})\right| \zeta_{(B \bar{B})_{1}}\right\rangle=0
\end{aligned}
$$

Hence, as the light field configuration $\left|\zeta_{B \bar{B}}\right\rangle_{1}$ does not couple to $\left|\zeta_{b \bar{b}}\right\rangle$ the physical system is equivalent to two decoupled subsystems, one containing $b \bar{b}$ and $(B \bar{B})_{0}$ components and the other containing the $(B \bar{B})_{1}$ component. Actually, $(B \bar{B})_{0}$ and $(B \bar{B})_{1}$ correspond to an isosinglet and to the neutral projection of an isotriplet respectively. Therefore, the lack of mixing between $\left|\zeta_{b \bar{b}}\right\rangle$, associated to the $b \bar{b}$ isosinglet, and $\left|\zeta_{B \bar{B}}\right\rangle_{1}$ is just a consequence of the isospin conservation in strong interactions.

These results make clear that the effect of two degenerate thresholds such as $B^{+} B^{-}$and $B^{0} \bar{B}^{0}$ can be taken into account through one effective $(B \bar{B})_{0}$ threshold whose mixing potential with $b \bar{b}$ contains an additional factor $\sqrt{2}$ as compared to that for a single threshold as $B_{s} \bar{B}_{s}$.
[1] N. Brambilla, S. Eidelman, C. Hanhart, A. Nefediev, C.-P. Shen, C. E. Thomas, A. Vairo, and C.-Z. Yuan, The $X Y Z$ states: Experimental and theoretical status and perspectives, Phys. Rep. 873, 1 (2020).

[2] K. J. Juge, J. Kuti, and C. J. Morningstar, Ab Initio Study of Hybrid $\bar{b} g b$ Mesons, Phys. Rev. Lett. 82, 4400 (1999).

[3] G. S. Bali, QCD forces and heavy quark bound states, Phys. Rep. 343, 1 (2001).

[4] G. S. Bali, H. Neff, T. Düssel, T. Lippert, and K. Schilling (SESAM Collaboration), Observation of string breaking in QCD, Phys. Rev. D 71, 114513 (2005).

[5] G. S. Bali, S. Collins, and C. Ehmann, Charmonium spectroscopy and mixing with light quark and open charm states from $n_{F}=2$ lattice QCD, Phys. Rev. D 84, 094506 (2011).

[6] J. Bulava, B. Hörz, F. Knechtli, V. Koch, G. Moir, C. Morningstar, and M. Peardon, String breaking by light and strange quarks in QCD, Phys. Lett. B 793, 493 (2019).

[7] E. Braaten, C. Langmack, and D. H. Smith, Born-Oppenheimer approximation for the $X Y Z$ mesons, Phys. Rev. D 90, 014044 (2014).

[8] R. Bruschini and P. González, Diabatic description of charmoniumlike mesons, Phys. Rev. D 102, 074002 (2020).

[9] F.-K. Guo, C. Hanhart, U.-G. Meißner, Q. Wang, Q. Zhao, and B.-S. Zou, Hadronic molecules, Rev. Mod. Phys. 90, 015004 (2018).
[10] R. F. Lebed, R. E. Mitchell, and E. S. Swanson, Heavyquark QCD exotica, Prog. Part. Nucl. Phys. 93, 143 (2017).

[11] A. Esposito, A. Pilloni, and A. Polosa, Multiquark resonances, Phys. Rep. 668, 1 (2017).

[12] H.-X. Chen, W. Chen, X. Liu, and S.-L. Zhu, The hiddencharm pentaquark and tetraquark states, Phys. Rep. 639, 1 (2016).

[13] M. B. Voloshin, Charmonium, Prog. Part. Nucl. Phys. 61, 455 (2008).

[14] R. Bruschini and P. González, Diabatic description of charmoniumlike mesons. II. Mass corrections and strong decay widths, Phys. Rev. D 103, 074009 (2021).

[15] E. J. Eichten, K. Lane, and C. Quigg, Charmonium levels near threshold and the narrow state $X(3872) \rightarrow \pi^{+} \pi^{-} J / \psi$, Phys. Rev. D 69, 094019 (2004).

[16] E. J. Eichten, K. Lane, and C. Quigg, New states above charm threshold, Phys. Rev. D 73, 014014 (2006); Erratum, Phys. Rev. D 73, 079903 (2006).

[17] S. L. Olsen, A new hadron spectroscopy, Front. Phys. (Beijing) 10, 121 (2015).

[18] P. Bicudo, M. Cardoso, N. Cardoso, and M. Wagner, Bottomonium resonances with $I=0$ from lattice QCD correlation functions with static and light quarks, Phys. Rev. D 101, 034503 (2020).

[19] P. Bicudo, N. Cardoso, L. Müller, and M. Wagner, Computation of the quarkonium and meson-meson composition of the $\Upsilon(n S)$ states and of the new $\Upsilon(10753)$ Belle resonance from lattice QCD static potentials, Phys. Rev. D 103, 074507 (2021). 
[20] P. A. Zyla et al. (Particle Data Group), Review of particle physics, Prog. Theor. Exp. Phys. 2020, 083C01 (2020).

[21] E. J. Eichten and C. Quigg, Mesons with beauty and charm: Spectroscopy, Phys. Rev. D 49, 5845 (1994).

[22] E. Eichten, K. Gottfried, T. Kinoshita, K. D. Lane, and T. M. Yan, Charmonium: The model, Phys. Rev. D 17, 3090 (1978); Erratum, Phys. Rev. D 21, 313 (1980).

[23] R. Bruschini and P. González (to be published).

[24] R. Bruschini and P. González, A plausible explanation of $\Upsilon(10860)$, Phys. Lett. B 791, 409 (2019).

[25] E. Eichten, S. Godfrey, H. Mahlke, and J. L. Rosner, Quarkonia and their transitions, Rev. Mod. Phys. 80, 1161 (2008).
[26] A. M. Badalian, B. L. G. Bakker, and I. V. Danilkin, Dielectron widths of the $S$-, $D$-vector bottomonium states, Phys. At. Nucl. 73, 138 (2010).

[27] E. Eichten and F. Feinberg, Spin-dependent forces in quantum chromodynamics, Phys. Rev. D 23, 2724 (1981).

[28] E. van Beveren, G. Rupp, T. A. Rijken, and C. Dullemond, Radial spectra and hadronic decay widths of light and heavy mesons, Phys. Rev. D 27, 1527 (1983).

[29] K. Heikkilä and N. A. Törnqvist, and S. Ono, Heavy $c \bar{c}$ and $b \bar{b}$ quarkonium states and unitarity effects, Phys. Rev. D 29, 110 (1984).

[30] J. Ferretti and E. Santopinto, Higher mass bottomonia, Phys. Rev. D 90, 094022 (2014). 